\title{
The Evolution of the Large-Scale Tail of Primordial Magnetic Fields
}

\author{
Karsten Jedamzik \\ Laboratoire de Physique Theorique et Astroparticules, UMR520\%-CNRS, \\ Universite Montpellier II, F-34095 Montpellier, France \\ Günter Sigl \\ II. Institut für Theoretische Physik, Universität Hamburg, \\ Luruper Chaussee 149, D-22761 Hamburg, Germany
}

\begin{abstract}
Cosmic magnetic fields may be generated during early cosmic phase transition, such as the QCDor electroweak- transitions. The magnitude of the remainder of such fields at the present epoch crucially depends on the exponent $n$ of their (initially super-Hubble) large-scale tail, i.e. $B_{\lambda} \sim \lambda^{-n}$. It has been claimed that causality requires $n=5 / 2$, contrary to much earlier claims of $n=3 / 2$. Here we analyze this question in detail. First, we note that contrary to current belief, the largescale magnetic field tail is not established at the phase transition itself, but rather continuoulsy evolves up to the present epoch. Neglecting turbulent flows we find $n=7 / 2$, i.e. very strongly suppressed large-scale fields. However, in the inevitable presence of turbulent flows we find that the large-scale magnetic field tail has sufficient time to evolve to that of the fluid turbulence. For white noise fluid turbulence this yields $n=3 / 2$ up to a certain scale and $n=5 / 2$ beyond for the magnetic field spectrum. This picture is also not changed when primordial viscosity and fluid flow dissipation is taken into account. Appreciable primordial magnetic fields originating from cosmic phase transitions seem thus possible.
\end{abstract}

\section{INTRODUCTION}

The origin of galactic- and cluster- magnetic fields is still unknown (cf. [1]). For long, one viable possibility was the generation of magnetic fields during some early magnetogenesis epoch [2] which than could act as seed fields for a galactic dynamo [3], or even under optimistic conditions provide the complete magnetic field after gravitational collapse 44. Another viable option is the generation of magnetic fields in stars and the expulsion of these fields into the intergalactic medium after the end of the life time of stars [5]. In either scenario many open questions remain. Irrespective of the origin of galactic magnetic fields it is interesting to know if substantial primordial relic magnetic fields could have survived the evolution of the early Universe.

This is particularly so due to recent claims of a lower limit on the magnitude of magnetic field strength in the intergalactic medium of $B \gtrsim 10^{-16}-10^{-14}$ Gauss (depening on coherence length scale) [6]. These claims are due to $\gamma$-ray observations of distant blazars by Fermi and HESS and detailed analysis of $\gamma$-ray showers on the extragalactic- and cosmic microwave- background radiation in the presence and absence of intergalactic magnetic fields (cf. 7]). They seem to indicate that a large volume fraction $f \gtrsim 0.5$ of voids a filled by magnetic fields which seems only difficult to achieve in scenarios of outflows/winds from galaxies. [8]

It has been claimed [9], that phase transition generated primordial magnetic [10] fields are too weak to be at the origin of the observed galactic- and cluster- magnetic fields, even under very optimistic assumptions on galactic dynamo amplification. The only exception would be here the case of completely helical fields produced during the QCD transition, nevertheless, requiring substantial dy- namo amplification as well [11]. The main point here is that due to "causality" arguments, it is claimed, the spectral index $n$ in

$$
\epsilon_{B}(k) \simeq \frac{1}{8 \pi} \int_{0}^{k} \mathrm{~d}^{3} k^{\prime} \mathbf{B}_{\mathbf{k}}^{*} \mathbf{B}_{\mathbf{k}}=\epsilon_{B}^{0}\left(\frac{k}{k_{0}}\right)^{2 n}
$$

would be large $n \gtrsim 5 / 2$ yielding a very blue spectrum and not much power on scales much above the phase transition Hubble radius. Here $\mathbf{B}_{\mathbf{k}}$ is the Fourrier transform of magnetic field, $k$ is wave vector, and $\epsilon_{B}$ is magnetic energy density.

In this paper we analyze the question of the spectral index in detail. In the next subsection we compute the magnetic field tail arising from the superposition of uncorrelated magnetic dipoles in vaccum, exemplifying that $n=3 / 2$ [12 is possible even for supper-Hubble distances. In Section III we replace the vacuum by the highly conductive medium in the early Universe, finding a suppressed $n=7 / 2$. In Section IV we include the realistic existence of fluid flows in our analysis analyzing the evolution of the large-scale tail first, in the turbulent fluid, and then in a fluid described by alternating periods of turbulent and viscous magnetohydrodynamics (MHD), as shown to occur in Ref [13]. In both cases we find $n=3 / 2$ up to some large scale. Conclusions are drawn in Section V. 


\section{THE MAGNETIC FIELD SPECTRUM DUE TO UNCORRELATED DIPOLES}

For a magnetic dipole density $\mathbf{M}$, in the magneto-static approximation the magnetic field can be written as

$\mathbf{B}(\mathbf{r}) \propto \int d^{3} \mathbf{r}^{\prime} \frac{3\left(\mathbf{r}-\mathbf{r}^{\prime}\right) \mathbf{M}\left(\mathbf{r}^{\prime}\right) \cdot\left(\mathbf{r}-\mathbf{r}^{\prime}\right)-\mathbf{M}\left(\mathbf{r}^{\prime}\right)\left|\mathbf{r}-\mathbf{r}^{\prime}\right|^{2}}{\left|\mathbf{r}-\mathbf{r}^{\prime}\right|^{5}}$,

where $\mu_{0}$ is the magnetic susceptibility in vacuum. From this one can easily show that

$$
\left\langle\mathbf{B}_{\mathbf{k}} \mathbf{B}_{-\mathbf{k}}\right\rangle \propto \int d^{3} \mathbf{r}^{\prime}\left\langle\mathbf{M}(\mathbf{r}) \mathbf{M}\left(\mathbf{r}^{\prime}\right)\right\rangle e^{i \mathbf{k} \cdot\left(\mathbf{r}-\mathbf{r}^{\prime}\right)}
$$

For uncorrelated dipoles, $\left\langle\mathbf{M}(\mathbf{r}) \mathbf{M}\left(\mathbf{r}^{\prime}\right)\right\rangle \propto \delta\left(\mathbf{r}-\mathbf{r}^{\prime}\right)$, this gives $\left\langle\mathbf{B}_{\mathbf{k}} \mathbf{B}_{-\mathbf{k}}\right\rangle=$ const and therefore, comparing with Eq. (1), a slope $\epsilon_{B}(k) \propto k^{3}$, thus $n=3 / 2$. The magnetostatic approximation is good at scales much smaller than the light-travel distance and thus certainly at Mpc scales, which are much smaller than the Hubble radius today.

\section{MAGNETIC FIELD EVOLUTION NEGLECTING FLUID FLOWS}

We assume that the total electrical current density consists of an external source $\mathbf{j}_{\text {ex }}$ and an Ohmic current induced by the electro-magnetic field,

$$
\mathbf{j}=\mathbf{j}_{\mathrm{ex}}+\sigma(\mathbf{E}+\mathbf{v} \times \mathbf{B})
$$

with $\sigma$ the conductivity. Here the non-vanishing external current $\mathbf{j}_{\text {ex }}$ is assumed to be dynamically forced during the epoch of magnetogenesis. Upon neglecting the displacement current $\partial_{t} \mathbf{E}$ for the total current one has Ampères law $4 \pi \mathbf{j}=\nabla \times \mathbf{B}$. Writing the external current as the rotation of the dipol-density $\mathbf{M}$,

$$
\mathbf{j}_{\mathrm{ex}}=\nabla \times \mathbf{M}
$$

inserting E from Eq. (3) into Faraday's induction equation

$$
\partial_{t} \mathbf{B}=-\nabla \times \mathbf{E}
$$

and using $\boldsymbol{\nabla} \cdot \mathbf{B}=0$ one has

$$
\partial_{t} \mathbf{B}=\boldsymbol{\nabla} \times(\mathbf{v} \times \mathbf{B})+\eta[\Delta \mathbf{B}-4 \pi(\Delta \mathbf{M}-\boldsymbol{\nabla}(\boldsymbol{\nabla} \cdot \mathbf{M}))],
$$

where we have used the resistivity $\eta=1 /(4 \pi \sigma)$.

We now express these equations in the expanding Universe in terms of redshift $z$ and at the same time perform a Fourier transformation of the spatial dependence. Using $\partial_{z}=-1 /[(1+z) H(z)] \partial_{t}$ with $H=\dot{a} / a$ the Hubble constant and the scale factor $a=1 /(1+z)$ and neglecting the fluid velocity term in Eq. (6) one then obtains for the evolution of the Fourier component $\mathbf{B}_{\mathbf{k}}(z)$ for comoving wave number $\mathbf{k}$,

$$
\begin{aligned}
\partial_{z} \mathbf{B}_{\mathbf{k}}(z)= & 2 \frac{\mathbf{B}_{\mathbf{k}}(z)}{1+z}+ \\
& +\eta(z) \frac{1+z}{H(z)} \mathbf{k}^{2}\left[\mathbf{B}_{\mathbf{k}}(z)-4 \pi \mathbf{M}_{\mathbf{k}}^{\perp}(z)\right],
\end{aligned}
$$

where $\mathbf{M}_{\mathbf{k}}^{\perp}(z) \equiv \mathbf{M}_{\mathbf{k}}(z)-\left(\hat{\mathbf{k}} \cdot \mathbf{M}_{\mathbf{k}}(z)\right) \hat{\mathbf{k}}$ is the component of $\mathbf{M}_{\mathbf{k}}(z)$ perpendicular to $\mathbf{k}$, with $\hat{\mathbf{k}}$ the unit vector in direction of $\mathbf{k}$. The solution of Eq. (7) which vanishes before the source term is switched on is given by

$$
\mathbf{B}(\mathbf{k}, z)=4 \pi \mathbf{k}^{2}(1+z)^{2} \int_{z}^{\infty} \exp \left[-\mathbf{k}^{2} \int_{z}^{z^{\prime}} \frac{\eta(\tilde{z})(1+\tilde{z})}{H(\tilde{z})} d \tilde{z}\right] \frac{\eta\left(z^{\prime}\right) \mathbf{M}_{\perp}\left(\mathbf{k}, z^{\prime}\right)}{\left(1+z^{\prime}\right) H\left(z^{\prime}\right)} d z^{\prime}
$$

It is convenient to define a critical wave number as

$$
k_{r}(z)=\left[\int_{z}^{\infty} \frac{\eta(\tilde{z})(1+\tilde{z})}{H(\tilde{z})} d \tilde{z}\right]^{-1 / 2} .
$$

For $k \gg k_{r}$ the exponential in Eq. (8) cuts off contributions from $z+\Delta z \gtrsim z+H(z) /\left[k^{2}(1+z) \eta(z)\right]$ so that one obtains

$$
\mathbf{B}_{\mathbf{k}}(z) \simeq 4 \pi \mathbf{M}_{\mathbf{k}}^{\perp}(z)
$$

This is the small-scale instantaneous limit in which the field is given by the superposition of dipole fields which are only subject to redshift since the second term in Eq. (7) approximately vanishes. In the opposite limit, $k \ll k_{r}$, the exponential in Eq. (8) can be set to one and one obtains

$$
\begin{aligned}
\mathbf{B}_{\mathbf{k}}(z) & \simeq 4 \pi \mathbf{k}^{2}(1+z)^{2} \int_{z}^{\infty} \frac{\eta\left(z^{\prime}\right) \mathbf{M}_{\mathbf{k}}^{\perp}\left(z^{\prime}\right)}{\left(1+z^{\prime}\right) H\left(z^{\prime}\right)} d z^{\prime} \\
& \simeq 4 \pi\left[\frac{k}{k_{r}(z)}\right]^{2} \mathbf{M}_{\mathbf{k}}^{\perp}(z)
\end{aligned}
$$

where in the second estimate we have used that

$$
\mathbf{M}_{\mathbf{k}}^{\perp}\left(z^{\prime}\right) \simeq\left(\frac{1+z^{\prime}}{1+z}\right)^{2} \mathbf{M}_{\mathbf{k}}^{\perp}(z)
$$

In this limit the resulting field is thus frozen into the plasma and strongly suppressed compared to the source 
term by a factor $\left(k / k_{r}\right)^{2}$ due to the screening by the conductivity of the medium. It is easy to see that the critical wave number becomes smallest at late times, when electrical conductivity and Hubble constant become smallest. Using the Spitzer resistivity $\eta \simeq \pi m_{e}^{1 / 2} e^{2} / T^{3 / 2}$ [14] one finds

$$
k_{r}(T) \simeq 3 \times 10^{3}\left(\frac{T}{T_{0}}\right)^{1 / 2} \mathrm{pc}^{-1} \text { for } T \lesssim 1 \mathrm{eV},
$$

where $T_{0}$ is the present day CMBR temperature. This should be compared to, for example, the comoving QCD Hubble radius wave vector, $k_{\mathrm{QCD}} \approx 6 \mathrm{pc}^{-1}(T / 100 \mathrm{MeV})$, showing that screening of the plasma is extremely efficient, leading only to highly suppressed magnetic fields on large scales. The supression here, $n=7 / 2$ is substantially larger that that claimed to apply in Ref. [9]. However, we will see in the next section that the conclusions change drastically when fluid motions are taken into account.

\section{MAGNETIC FIELD EVOLUTION WITH FLUID FLOWS}

As post-inflationary "causal" magnetogenesis in the early Universe most likely takes place during cosmic firstorder phase transitions, and during such transitions the generation of cosmic turbulence is inevitable, the complete neglect of fluid motions is thus unrealistic. In the one-fluid approximation of magnetohydrodynamics (MHD) the velocity field $\mathbf{v}$ evolves according to

$$
\partial_{t} \mathbf{v}+(\mathbf{v} \cdot \boldsymbol{\nabla}) \mathbf{v}=\frac{(\boldsymbol{\nabla} \times \mathbf{B}) \times \mathbf{B}}{4 \pi \rho}+\mathbf{f}_{v},
$$

where $\rho$ is the fluid density and $\mathbf{f}_{v}$ is the viscous damping force [13]. We assume now that the fluid kinetic energy density (at least initially) dominates magnetic energy density. Furthermore we assume that the phase transition induced magnetization $\mathbf{M}$ has led to the build-up of some magnetic seed field on relatively small scales (cf. Sec. III), but nothing on large scales. We are then interested to know how much magnetic field will be generated on large scales due to non-linear interactions between magnetic- and velocity- fields on smaller scales. The evolution of magnetic fields are given by

$$
\partial_{t} \mathbf{B}=\boldsymbol{\nabla} \times(\mathbf{v} \times \mathbf{B})+\eta \Delta \mathbf{B} .
$$

We are not interested in the detailed magnetic field evolution, but rather in the average buildup of magnetic energy density taken over an ensemble of cosmic realizations, i.e.

$$
\left\langle\partial_{t} M_{k}\right\rangle
$$

with $M_{k}$ the magnetic spectral energy (not to be confused with the magnetisation) defined through

$$
\epsilon_{B}=\frac{1}{8 \pi V} \int \mathrm{d}^{3} x \mathbf{B}^{2}(x) \equiv \int \mathrm{d} k M_{k}
$$

where $V$ denotes volume and where we have implicitly assumed cosmic homogeneity and isotropy rendering $M_{k}$ a function only of the magnitude of wavevector $\mathbf{k}$.

Ensemble averaging over the product of Eq. (15) and B, assuming time-independent and uncorrelated fluid turbulence, i.e. $\left\langle v_{\mathrm{k}}^{*}(t) v_{\mathrm{k}^{\prime}}\left(t^{\prime}\right)\right\rangle \sim \delta_{\mathbf{k}^{\prime} \mathbf{k}} \delta_{t^{\prime} t}$ unmodified by the magnetic fields, i.e. neglecting the backreaction magnetic force term in Eq. 114. Such a calculation has been performed first by Ref. 15. We follow here the analysis of Ref. [16] which leads to the following evolution equation for $M_{k}$ [17]

$$
\frac{\partial M_{q}}{\partial t} \simeq \mathcal{C} \int \mathrm{d} k \mathrm{~d} \theta \frac{q^{4}}{k k_{1}^{3}} \frac{v^{2}\left(L_{k_{1}}\right)}{v\left(L_{k}\right)} f(q / k, \theta) M_{k},
$$

where for simplicity we have dropped the ensemble average brackets and discarded terms of higher order in wavevector $\mathbf{k}$ as well as terms containing magnetic helicity. In other words, we are only interested in the small $k$ tail of non-helical fields. Eq. (18) describes the generation of magnetic energy on scale with wave vector $\mathbf{q}$ by the non-linear interaction between magnetic modes with wave vector $\mathbf{k}$ and velocity modes with wave vectors $\mathbf{k}_{\mathbf{1}} \equiv \mathbf{q}-\mathbf{k}$. Here $\theta$ is the angle between $\mathbf{q}$ and $\mathbf{k}$, $v\left(L_{k}\right)$ are typical velocities on scale $L_{k}=2 \pi / k$ associated with wave vector $k$, and $\mathcal{C}$ is a crucial constant estimated approximately as $\mathcal{C} \sim(2 \pi)^{2}$. Furthermore, the function $f$ is given by

$$
f(x, \theta)=\sin ^{3}(\theta) \frac{\left(1+x^{2}-x \cos \theta\right)}{\left(1+x^{2}-2 x \cos \theta\right)},
$$

where the denominator is $k_{1} / k$. We now assume a power law for the scale dependence of the fluid velocity, i.e.

$$
v^{2}\left(L_{k}\right)=v_{g}^{2}\left(\frac{L_{0}}{L_{k}}\right)^{\alpha}=v_{g}^{2}\left(\frac{k}{k_{0}}\right)^{\alpha},
$$

where $L_{0}=2 \pi / k_{0}$ is some reference scale on which $v=v_{g}$. Here $\alpha=3$ corresponds to uncorrelated white noise whereas $\alpha=-2 / 3$ corresponds to a red Kolmogoroff spectrum. Inserting Eq. (20) into Eq. (18), changing to comoving wave vectors $k$ with $k_{\text {phys }}=k / a$, where $a$ denotes the scale factor, one finds

$$
\frac{\partial M_{q}}{\partial \ln a} \simeq \mathcal{C} \frac{a}{H_{g}} \int_{q}^{k_{I}(a)} \mathrm{d} \ln k k\left(\frac{q}{k}\right)^{4} v_{g}\left(\frac{k}{k_{0}}\right)^{\alpha / 2} G^{\alpha}\left(\frac{q}{k}\right) M_{k}
$$

for the evolution of magnetic energy density during a radiation dominated Universe. Here $k_{I}(a)$ is some effective ultraviolet cutoff, or damping scale, to be discussed below, and $H_{g}$ is the Hubble constant at the initial magnetogenesis era where we have chosen scale factor $a=1$. Note that equation Eq. (21) really holds for some properly defined comoving $M_{k}$ which takes into account the redshifting $\epsilon_{B} \sim 1 / a^{4}$ of magnetic energy density [18]. During matter domination the dependence on the scale factor of Eq. (21) is changed from $a$ to $a^{1 / 2}$. The function 
$G^{\alpha}$ in Eq. (21) contains the $\theta$ integration

$$
G^{\alpha}(x)=\int_{0}^{\pi} \mathrm{d} \theta \sin ^{3}(\theta) \frac{\left(1+x^{2}-x \cos \theta\right)}{\left(1+x^{2}-2 x \cos \theta\right)^{5 / 2-\alpha / 2}}
$$

We are only interested in large-scale modes $q \lesssim k$. In this case $G^{\alpha}(x)$ is a slowly varying function, with, for example $G^{3}$ varying only from $4 / 3$ to $5 / 3$ when $x$ goes from 0 to 1. We therefore conservatively drop it.

Eq. (18) represents our master equation. It demonstrates that magnetic energy may be transferred from small scales (large $k$ ) to large scales (small $q$ ) via nonlinear processes between fluid flows and magnetic fields, and that this process apparently becomes more efficient at later epochs (larger $a$ ). Before presenting a prediction for the evolution of the large-scale tail of magnetic fields, we have to emphasize a conceptual difference between the findings of this work and all other prior studies of primordial magnetic fields. So far, it had been believed that the epochs of magnetogenesis and subsequent field decay are well separated. In other words, it would be assumed that some magnetogenesis scenario during a cosmic phase transition would fully account for all field properties, in particular, also those on large scales, and that the final late time field would be simply given by all magnetic energy which hadn't been dissipated yet. This study shows that magnetogenesis should be considered as a continous process, which in particular holds true for the large-scale tail, with magnetic fields generated on small $q$ even long after the epoch of the initial magnetogenesis era.

We now proceed to estimate the growth of large-scale magnetic fields. We assume that the kinetic energy density is peaked on some scale $k_{I}$, which we refer to as the integral scale. For $k>k_{I}$ the velocity spectrum is red (e.g. Kolmogoroff), and for $k<k_{I}$ given by Eq. (20). Contributions from $v$ modes with $k>k_{I}$ to the integral Eq. (18) may be approximatively neglected, as already done in Eq. (21). We assume that the magnetic seed field only exists on some small scale $k_{B}$, with $M_{q}=0$ for either $q \ll k_{B}$ and $q \gg k_{B}$. For the moment we make the crucial assumption that $k_{B} \sim k_{I}$ and that equipartition holds on that scale, i.e. $M_{k} \approx E_{k}$, with $\epsilon_{K} \equiv \int \mathrm{d} k E_{k}$, where $\epsilon_{K}$ is the kinetic energy density. We will discuss this assumption in some more detail below.

We need a model of the evolution of fluid flows in the expanding Universe, as this modifies the $v\left(L_{k}\right)$ entering Eq. (21). Such a model has been presented, in detail, in Ref. [13]. It had been found that the evolution of velocity and magnetic fields in the early Universe is quite complex, described by an alternation of turbulent phases and viscous phases. Here, early on, viscosity is due to neutrinos, and at later stages, due to photons. Nevertheless, we focus first on turbulent phases.

\section{A. Turbulent Phases}

In the absence of further turbulence producing mechanisms, turbulent flows simply decay when the eddy turnover rate equals the Hubble rate, i.e.

$$
\frac{v^{p}\left(k_{I}\right)}{L_{I}^{p}}=\frac{v^{p}\left(k_{I}\right) k_{I}^{p}}{2 \pi} \simeq H
$$

In particular, eddies on $k_{I}$ break up into eddies of larger $k$, with their energy getting transported to the dissipative scale $k_{\text {diss }}>k_{I}$ where it is converted to heat. Here the superscript $p$ denotes proper quantities. These are related to comoving quantities via $L^{p}=L a, k^{p}=k / a$, as well as $v^{p}=v$ (radiation dominated) and $v^{p}=v / a$ (matter dominated). From Eq. (23), using Eq. (20) and assuming radiation domination one derives

$$
k_{I}(a)=k_{I}^{0} a^{-2 /(\alpha+2)} .
$$

with

$$
k_{I}^{0}=\frac{2 \pi H_{g}}{v_{g}}
$$

the initial integral scale. Here we adopted the reference scale $k_{0}=k_{I}^{0}$ such that $\epsilon_{K}^{g}=\rho_{r}^{g} v_{g}^{2} / 2$ represents the initial turbulent energy density.

Assuming equipartition $M_{k}\left(k_{I}^{0}\right) \approx E_{k}\left(k_{I}^{0}\right)$ on the initial integral scale $k_{I}^{0}$ but $M_{k} \approx 0$ beyond, we are interested to know if the decaying magnetohydrodynamic turbulence may establish equipartition on even larger scales, and if yes, how large is the largest scale where equipartion may be achieved. Using Eq. (24), assuming equipartition $M_{k} \approx E_{k}$ on $k_{I}$ at all times (to be verified selfconsistently below), one may derive for the change in magnetic energy density $\Delta M_{q}(a)$ at redshift $a$

$$
\Delta M_{q}(a) \simeq \mathcal{C} \rho_{r}\left(\frac{q}{2 \pi H_{0}}\right)^{4} \frac{v_{g}^{7}}{H_{0}} a^{\frac{10-2 \alpha}{\alpha+2}},
$$

where $\rho_{r}$ is radiation energy density and where we neglected an unimportant numerical factor. The total change of $M_{q}$ is conservatively determined at $a_{q}$ where the integral scale has decreased to $q$, i.e. $k_{I}\left(a_{q}\right)=q$. The condition that by $a_{q}$ the change $\Delta M_{q}\left(a_{q}\right)>M_{q}^{\text {equi }}=$ $v_{g}^{2}\left(q / k_{I}^{0}\right)^{\alpha} / q$ is larger than the equipartition value then yields

$$
\mathcal{C} \gtrsim \frac{1}{2 \pi}
$$

independent (!) of $q, v_{g}, H_{g}$, and $\alpha$. As we determined $\mathcal{C} \approx(2 \pi)^{2}$ thus equipartition between magnetic fields and velocity fields may be attained on a large range of scales, independent of the turbulence spectrum. This justifies selfconsistently our above assumption.

At any late time, the magnetic field spectrum therefore has the form

$\frac{M_{q}(a)}{M_{I}(a)}= \begin{cases}{\left[\frac{q}{k_{I}(a)}\right]^{-\beta}} & k_{I}(a) \leq q \leq k_{\mathrm{diss}}(a) \\ {\left[\frac{q}{k_{I}(a)}\right]^{\alpha-1}} & k_{\text {equi }}(a) \leq q \leq k_{I}(a) \\ {\left[\frac{k_{\text {equi }}(a)}{k_{I}(a)}\right]^{\alpha-1}\left[\frac{q}{k_{\text {equi }}(a)}\right]^{4}} & q \leq k_{\text {equi }}(a),\end{cases}$ 
with the $q$-independent constant $M_{I}(a)=M_{k_{I}(a)}^{\text {equi }}$. This is Kolmogoroff $(\beta=2 / 3)$ or Ironshnikov-Kraichnan $(\beta=$ $1 / 2$ ) on very small scales, following the fluid spectrum on scales larger than the integral scale $k_{I}(a)$ and turning over to a steeper spectrum for $k<k_{\text {equi }}(a)$. Here $k_{\text {equi }}(a)$ is the smallest wavevector for which equipartition could be attained at redshift $a$. It is given by

$$
k_{\text {equi }}(a) \simeq(2 \pi \mathcal{C})^{\frac{1}{\alpha-5}} k_{I}(a) .
$$

The spectral index 4 for $k<k_{\text {equi }}$ in Eq. (28) is dictated by the power of $q$ in Eq. (21). The evolution of the spectrum for a representive case is displayed in Fig. [1. with the spectrum shown at temperatures $T=100 \mathrm{MeV}$, $T=100 \mathrm{keV}$, and $T=1 \mathrm{eV}$, respectively. Note that since the integral in the master equation (21) is dominated by the integral scale, these results are insensitive to the turbulence power spectrum at scales $k \ll k_{I}(a)$, and thus in particular on whether this spectrum is causal or not.

Eq. (24) and Eq. (26) apply only during radiation domination. It is easy to show [13] that during matter domination $k_{I}(a)$ stays constant or at best decreases logarithmically with scale factor. Using this plus the matterdomination modified Eq. (21), as well as taking into account of the proper redshifting of $v$, it can be shown that no further substantial growth of $M_{q}$ occurs during matter-domination. This holds true even when $v \approx v_{A}$, as should be the case, yielding a milder redshift dependence $v \sim a^{-1 / 2}$ than the above stated $v \sim a^{-1}$. Here $v_{A}$ is the Alfven velocity. This implies that $k_{\text {equi }}$ gets "frozen in" at the transition of radiation to matter domination at its minimum value $k_{\text {equi }}^{\mathrm{min}}$.

We comment here shortly on one implicit assumption made. In particular, we allow $M_{q}$ only to grow to its scale-dependent equipartition value $M_{q} \approx E_{q}$. Though this seem fairly plausible, it is not clear if, in fact, it is true. Rather, it is possible that on scales $q<k_{I}$ the magnetic field grows to super-equipartition, i.e. $M_{q}>E_{q}$. This may be possible as long as global energy conservation is not violated. It is not clear if this could happen, and only an improved analysis taking account of potential modifications of Eq. (18) due to backreaction of magnetic fields on fluid flows, as well as deriving an independent equation for $\left\langle\partial_{t} E_{k}\right\rangle$, could conclusively adress this issue. This formidable task is however beyond the scope of this paper. We note that our assumption $M_{q} \lesssim E_{q}$ is conservative.

\section{B. Viscous Phases}

A realistic calculation has to take into account the various small Reynolds number $R<1$ viscous phases of magnetohydrodynamics in the early Universe. Here viscous forces in Eq. (14) are given by [13]

$$
\mathbf{f}_{v}=\left\{\begin{array}{ll}
\eta \nabla^{2} \mathbf{v} & l_{\mathrm{mfp}} \ll L \\
-\alpha \mathbf{v} & l_{\mathrm{mfp}} \gg L
\end{array},\right.
$$
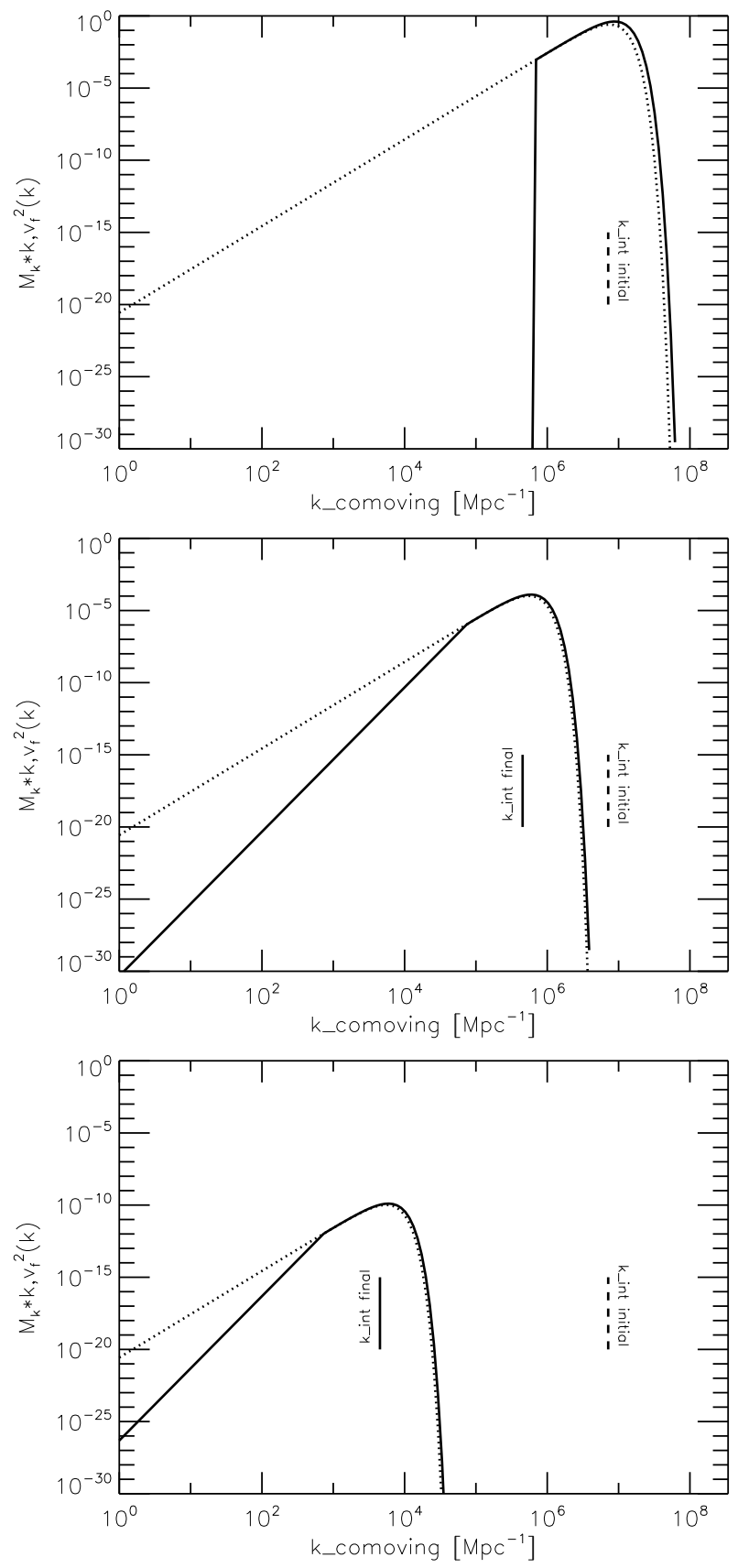

FIG. 1: Evolution of the magnetic energy spectrum (in units of the radiation energy density) according to Eq. (21) for a magnetogenesis temperature $T=100 \mathrm{MeV}$ and for $v_{g}=1$ and $\alpha=3$. The upper panel shows the assumed initial condition for the magnetic- (solid) and kinetic- (dotted) energy spectrum at $T=100 \mathrm{MeV}$, whereas the middle and lower panels show these quantities evolved to $T=100 \mathrm{keV}$ and $T=1 \mathrm{eV}$, respectively. Dashed and solid vertical line represent the integral scale at the initial and current temperature, respectively. The scale at which the solid line departs from the dotted line, is the scale $k_{\text {equi }}$ from Eq. (29) which in the present case reads $k_{\text {equi }}(a) \simeq 0.063 k_{I}(a)$. This is nicely confirmed by the simulation. Note that the semianalytic simulation assumes a turbulent regime throughout. 
where $l_{\mathrm{mfp}}$ is the mean free path of the momentum transporting particles, early on neutrinos, and later photons, and $L$ is the scale under consideration. It can be shown that the diffusion constant $\eta$ and drag constant $\alpha$ are essentially $\eta \simeq l_{\mathrm{mfp}}$ and $\alpha \simeq 1 / l_{\mathrm{mfp}}$ up to numerical, and statistical weight factors. Starting at high temperatures, as the mean free path of neutrinos increases with the expansion of the Universe, an initially turbulent fluid becomes viscous due to neutrino diffusion. At this point all pre-existing fluid motions will be erased up to the neutrino diffusion length, i.e. $d \simeq \sqrt{\eta t}$ with $t$ cosmic time. However, importantly, magnetic fields are not erased, and remain frozen into the plasma. On the other hand build-up of large-scale magnetic fields via Eq. (18) has stopped entirely as there are no appreciable fluid velocities. This viscous phase occurs already at fairly high temperatures $T \sim 20-80 \mathrm{MeV}$, depending on the kineticand magnetic- energies. As the neutrino mean free path increases beyond the scale $L_{I} \sim 1 / k_{I}$, the fluid continues its viscous period in the neutrino free-streaming regime. Form their on, the very small Reynolds number starts increasing again. Here secondary fluid velocities

$$
v \simeq \frac{k_{I} B^{2}}{\rho \alpha} \simeq \frac{v_{A}^{2}}{\alpha L_{c}}
$$

are generated via the magnetic stresses in Eq. (14). These velocities become larger and larger, and at some point, still in the viscous regime, the "instantaneous" dissipation scale $k_{\text {diss }}$ falls below the integral scale $k_{I}$ at the beginning of the viscous regime. At this point further magnetic- and fluid- energy density is dissipated leading to an increase of $k_{I} \approx k_{\text {diss }}$. Here $k_{\text {diss }}$ is given again by the condition Eq. (23) with velocities now as in Eq. (31). Somewhat later, typically at $T \sim 5-10 \mathrm{MeV}$ shortly before neutrino decoupling, the fluid becomes again fully turbulent. This cycle repeats itself at temperatures $T \lesssim 1 \mathrm{MeV}$, but now due to the long mean free path of photons.

It is known 13 that the net effect of theses viscous phases is a delay of the growth of the integral scale $L_{I}(a)$ during viscous periods themselves. Nevertheless, once turbulence has recommenced, the integral scale $L_{I}(a)$ is indistinguishable from $L_{I}^{\text {turb }}(a)$, the respective scale in a Universe which had always remained turbulent. This may be observed in Fig. 2, where results for $k_{I}(a)$ of a semi-analytic calculation of $L_{I}$ are shown. The simulation takes proper account of the correct neutrino- and photon- viscosities. It is far from clear if primordial viscosity has a similar effect on $L_{\text {equi }}(a)$ the maximum scale where local equipartition on large scales has been attained, in particular, simply the delay of growth of this quantity. During large parts of the viscous eras no growth of magnetic field energy density via Eq. (18) may occur. This could yield much weaker $M_{q}$. On the other hand, towards the end of the viscous periods $M_{k_{I}}$ entering Eq. (18) is genuinely larger than when viscosity would be small. It turns out that both effects roughly cancel each other as can be seen in Fig. 2, The detailed

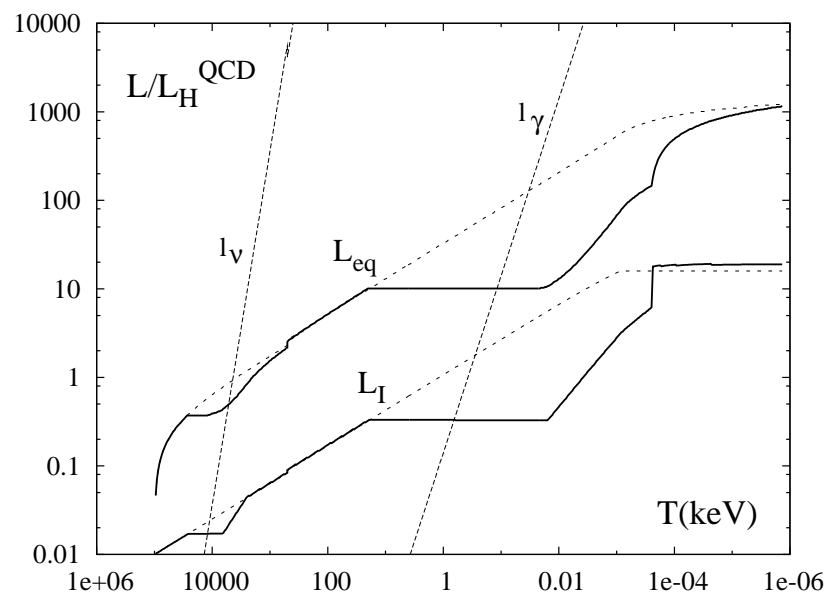

FIG. 2: Evolution of various length scales as a function of temperature. All length scales are in units of the approximate QCD Hubble radius, i.e. $L_{H}^{Q C D} \approx 1 \mathrm{pc}$. Shown are the integral scale $L_{I}$ on which most of the magnetic- and kinetic- energy is contained, as well as the equipartition scale $L_{\text {equi }}$, i.e. the largest scale on which equipartition between kinetic turbulent energy and magnetic energy could be attained. Each length scale is shown twice, once for a Universe which is always turbulent (dotted lines) and once for a realistic Universe, where turbulent and viscous MHD are alternating due to neutrino- and photon viscosity (solid lines). The dashed lines, show the neutrino- and photon- mean free path as labeled. The results of this semi-analytic simulation assume an initial magnetogenesis epoch at $T=100 \mathrm{MeV}$ with $\epsilon_{K}^{g}=10^{-4} \rho_{r}^{g}$ and $\epsilon_{B}^{g}=10^{-9} \rho_{r}^{g}$, where $\epsilon_{B}^{g}, \epsilon_{K}^{g}$, and $\rho_{r}^{g}$ are initial magnetic-, kinetic-, and total radiation energy density, respectively. A white noise $\alpha=3$ spectrum has been assumed for the slope of the turbulence on large scales $L \gtrsim L_{I}$.

simulation shows that as in the case of $k_{I}(a), k_{\text {equi }}(a)$ during turbulent phases is as if viscous phases had never occurred.

We now briefly comment on the adopted initial conditions of equipartition on $k_{I}$, though we do not intend to go into much detail of the initial magnetogenesis period itself. Using Eq. (18) one may derive an approximate equation $\partial M_{k_{I}} / \partial t \simeq M_{k_{I}} / \tau$ with $\tau$ given by $\tau^{-1} \approx \mathcal{C} v\left(k_{I}\right) k_{I}$ and thus is a fraction of the eddy turnover time on the integral scale. We note that since $v\left(k_{I}\right) k_{I} /(2 \pi) \gtrsim H$ the requirement to have more than one e-fold of amplification of magnetic fields on $k_{I}$ per Hubble time during the phase transition reads $\mathcal{C} \gtrsim 2 \pi$. Since this is the case, even initially much weaker than equipartion fields are likely to come to equipartion during the phase transition. Nevertheless, this does not adress concerns about possibly most magnetic energy going into large $q$ modes, due to the stronq $q^{4}$ dependence in Eq. (18). 


\section{Results}

Given all the above, the final present-day spectrum, barring further evolution in the non-linear structure formation regime, is therefore given by Eq. (28) with $k_{\text {equi }}$ and $k_{I}$ replaced by $k_{\text {equi }}^{\text {min }}$ and $k_{I}^{\mathrm{min}}$, their respective values at matter-radiation equality. In this subsection we deviate from our above chosen convention that $a=1$ at the initial magnetogenesis epoch. Rather, as customary, we choose $a=1$ at the present epoch. In this case $k_{I}^{\min }$ and $M_{I}\left(a_{e q}\right)$ in Eq. (28) are given by

$$
\begin{aligned}
k_{I}^{\min }= & k_{I}\left(a_{e q}\right)=\frac{2 \pi H_{g}}{v_{g}}\left(\frac{H_{e q}}{H_{g}}\right)^{\frac{\alpha+4}{2 \alpha+4}} a_{e q} \\
M_{I}\left(a_{e q}\right) & =\rho_{r} v_{g}^{2}\left(\frac{H_{e q}}{H_{g}}\right)^{\frac{2 \alpha}{\alpha+2}} \frac{1}{k_{I}\left(a_{e q}\right)},
\end{aligned}
$$

where $H_{e q}$ and $a_{e q} \approx 5 \times 10^{3}$ are Hubble constant and scale factor at matter radiation equality, respectively. We note that for $v_{g} \simeq 1$, i.e. the speed of light, their is equipartition between kinetic turbulent energy $\epsilon_{K}^{g}$ and radiation density $\rho_{r}^{g}$ (as well as subsequently magnetic energy density $\epsilon_{B}^{g}$ ). Such equipartion corresponds to $B_{0} \simeq 5.7 \times 10^{-6}$ Gauss in comoving magnetic field strength. Making the reasonable assumption that the turbulence is white noise (cf. [19] for a numerical justification), i.e. $\alpha=3$ yields spectral index $n=3 / 2$ for $k>k_{\text {equi }}$ and only for $k<k_{\text {equi }}$ the in Ref. [9] proposed $n=5 / 2$. We will see that this leads to strongly modified predictions for the possible large-scale magnetic field strength arising due to "causal" processes compared to those given in Ref. [9]. The scale $L_{\text {equi }}^{\min }=2 \pi / k_{\text {equi }}^{\min }$ may be obtained via Eq. (29) and Eq. (32)

$$
L_{\text {equi }}^{\min } \simeq 30 \mathrm{kpc}\left(\frac{\epsilon_{K}^{g}}{\rho_{r}^{g}}\right)^{1 / 2}\left(\frac{T_{g}}{100 \mathrm{MeV}}\right)^{-3 / 5} \quad \text { for } \alpha=3 \text {. }
$$

To derive an upper bound we assume the maximum plausible energy in the turbulence, i.e. equipartition $\epsilon_{K}^{g} \simeq \rho_{r}^{g}$ with the radiation energy density. We derive the magnetic field strength on scale $L$ from Eq. (28) and Eq. (32) - Eq. (33) taking account of the conversion given below Eq. (33). On $L \simeq 100 \mathrm{kpc}$, the scale chosen by Ref. 9] we find $B(100 \mathrm{kpc}) \lesssim 5 \times 10^{-14}$ Gauss for the QCD transition $\left(T_{g} \simeq 100 \mathrm{MeV}\right)$. This value is also reflected by Fig. 1, lower panel when using that $B_{k} \simeq 5.7 \times 10^{-6}\left(M_{k} k / \rho_{r}\right)^{1 / 2}$ Gauss. For the electroweak transition $\left(T_{g} \simeq 100 \mathrm{GeV}\right)$ one has $B(100 \mathrm{kpc}) \lesssim 3 \times$ $10^{-20}$ Gauss. This should be compared to the much smaller $B(100 \mathrm{kpc}) \lesssim 10^{-27}$ Gauss given in Ref. 9] for the electroweak transition.

However, we stress here again [4, 13] that more important than the magnetic field strength on some large scale $\sim 100 \mathrm{kpc}$ should be the total magnetic energy on all scales. Numerical simulations show [20] that gravitationally induced inverse cascades during cluster collapse seems to transfer this magnetic power to large scales.
We thus restate here [4, 13] the prediction for present day magnetic field strength and coherence length as a function of magnetogenesis temperature $T_{g}$, initial magnetic/turbulent energy density, $r_{g}$ (in units of radiation energy density), and initial helicity $h_{g}$ (as a fraction of maximal helicity)

$$
\begin{aligned}
& L_{c}(T) \simeq 12 \mathrm{pc}\left(\frac{r_{g}}{0.01}\right)^{1 / 2}\left(\frac{T_{g}}{100 \mathrm{GeV}}\right)^{-3 / 5} \\
& B_{c}(T) \simeq 6.0 \times 10^{-14} \mathrm{G}\left(\frac{r_{g}}{0.01}\right)^{1 / 2}\left(\frac{T_{g}}{100 \mathrm{GeV}}\right)^{-3 / 5}(35)
\end{aligned}
$$

for non-helical fields and

$$
\begin{aligned}
L_{c}(T) \simeq & 1.9 \mathrm{kpc}\left(\frac{r_{g}}{0.01}\right)^{1 / 2}\left(\frac{h_{g}}{0.01}\right)^{1 / 3}\left(\frac{T_{g}}{100 \mathrm{GeV}}\right)^{-1 / 3} \\
B_{c}(T) \simeq & 1.6 \times 10^{-11} \mathrm{G} \\
\times & \left(\frac{r_{g}}{0.01}\right)^{1 / 2}\left(\frac{h_{g}}{0.01}\right)^{1 / 3}\left(\frac{T_{g}}{100 \mathrm{GeV}}\right)^{-1 / 3},
\end{aligned}
$$

for helical fields. Here Eq. 35 assumes a spectral index $n=3 / 2$.

\section{CONCLUSIONS}

In this paper we have considered the large-scale (small wavenumber $k$ ) tail of primordial magnetic fields produced "causally" (i.e. not during an inflationary epoch) in the early Universe. Here we focussed on scales which are instantaneously super-Hubble during the initial magnetogenesis epoch, i.e. $\lambda \gtrsim 1$ pc, as such scales correspond to current galactic scales. It has been claimed [9] that due to causality such fields are highly supressed. i.e. $n=5 / 2$ in Eq. (1). We have shown here that this may not be the case.

It is customarily assumed that an initial period of magnetogenesis (e.g. during a phase transition) may be well separated from the subsequent epoch of freely decaying MHD. In other words, field production only happens during the magnetogenesis epoch, whereas subsequently only field dissipation takes place. However, this is not the case, magnetic field on large scales may be continously generated due to nonlinear interactions of small scale fluid- and magnetic- modes, even well after the initial epoch of magnetogenesis. We have shown that, with the expansion of the Universe, this process is rapid enough to yield scale-dependent equipartition between fluid velocities and magnetic fields on ever and ever larger scales. In particular, barring super-equipartion values of the magnetic field, this implies that the kinetic- and magneticenergy tail could have the same spectral index. As white noise $n=3 / 2$ for the fluid is expected [19], the magnetic field tail could also develop $n=3 / 2$.

When the realistic alternation of turbulent and viscous MHD (due to photon- and neutrino- fluid viscosity) in the early Universe is considered, the evolution becomes substantially more complicated. We have followed 
this with a semi-analytic treatment. During turbulent epochs the fluid forces equipartition with magnetic fields on small scales. After this, amplification of large-scale magnetic fields due to non-linear interactions between velocity- and magnetic- modes on small scales becomes important. When fluid viscosity becomes considerable, all fluid flows up to the neutrino (photon) diffusion scale become erased, and the process of amplification of largescale magnetic fields effectively stops. Only when neutrino (photon) interaction with the plasma become very weak, new fluid flows are generated by the priorly generated and frozen in magnetic fields. Thus the fluid initial kinetic energy had been stored in magnetic field energy. Subsequently further large-scale magnetic field amplification takes place. As complicated as is, the net result of these processes is a present day large-scale magnetic field tail essentially indistinguishable from that if the Universe would have stayed turbulent throughout.

However, we caution that our results are preliminary, as our evolution equation may not include all magnetic sink terms on large scales. Those were assumed to be- come important only at scale-dependent equipartition, i.e. when $M_{q} \approx E_{q}$. Furthermore, backreaction of magnetic fields on fluid flows was only taken into account in a heuristic way. A complete analysis, which is beyond the scope of the present paper, should be performed to confirm our results.

The resulting magnetic field spectrum may thus be $n=$ $3 / 2$ up to some large scale $L_{\text {equi }}^{\text {min }}$ in Eq. (34) and $n=5 / 2$ beyond. This would lead to considerably larger magnetic field strength on large scales than claimed in Ref. [9]. If appreciable primordial magnetic fields actually exist, however, can be verified only by measuring the magnetic field in the intergalactic medium.

Acknowledgments We thank Chiara Caprini, Torsten Ensslin, Ruth Durrer, Tina Kahniashvili, Kandu Subramanian, and Tanmay Vachaspati for useful conservations. This work was supported by the Deutsche Forschungsgemeinschaft through SFB 676 "Particles, Strings and the Early Universe: The Structure of Matter and SpaceTime".
[1] A. Brandenburg and K. Subramanian, Phys. Rept. 417, 1 (2005) arXiv:astro-ph/0405052.

[2] see, e.g., D. Grasso, H. R. Rubinstein, Phys. Rept. 348, 163-266 (2001). astro-ph/0009061.

[3] R. M. Kulsrud, Ann. Rev. Astron. Astrophys. 37, 37-64 (1999).

[4] R. Banerjee and K. Jedamzik, Phys. Rev. Lett. 91, 251301 (2003) [Erratum-ibid. 93, 179901 (2004)] arXiv:astro-ph/0306211.

[5] see, e.g., J. P. Vallee, Fundamentals of Cosmic Physics, 19, 1-89 (1997).

[6] A. Neronov and I. Vovk, Science 328, 73 (2010); F. Tavecchio, G. Ghisellini, G. Bonnoli and L. Foschini, arXiv:1009.1048 [astro-ph.HE].

[7] F. A. Aharonian, P. S. Coppi and H. J. Volk, Astrophys. J. 423, L5 (1994); R. Plaga, Nature 374, 430 (1995); A. Neronov and D. V. Semikoz, JETP Lett. 85, 473 (2007) arXiv:astro-ph/0604607, A. Elyiv, A. Neronov and D. V. Semikoz, Phys. Rev. D 80, 023010 (2009).

[8] K. Dolag, M. Kachelriess, S. Ostapchenko and R. Tomas, arXiv:1009.1782 [astro-ph.HE].

[9] C. Caprini and R. Durrer, Phys. Rev. D 65, 023517 (2001); R. Durrer and C. Caprini, JCAP 0311, 010 (2003); C. Caprini and R. Durrer, Phys. Rev. D 72, 088301 (2005).
[10] G. Sigl, A. V. Olinto and K. Jedamzik, Phys. Rev. D 55, 4582 (1997); G. Sigl, Phys. Rev. D 66, 123002 (2002).

[11] C. Caprini, R. Durrer and E. Fenu, JCAP 0911, 001 (2009).

[12] C. J. Hogan, Phys. Rev. Lett. 51, 1488 (1983).

[13] R. Banerjee and K. Jedamzik, Phys. Rev. D 70, 123003 (2004) arXiv:astro-ph/0410032.

[14] see, e.g., R. Choudhuri, The Physics of Fluids and Plasmas: An Introduction for Astrophysicists (Cambridge University Press, 1999).

[15] S. I. Vainshtein, Soviet Phys. JETP, 31, 87 (1970).

[16] R. M. Kulsrud and S. W.Anderson, Astrophys. J. 396, 606 (1992).

[17] The equation follows from Ref. 's Eqs. (2.30) (with only the first term on the RHS), Eqs. (2.31), (2.33), and (2.34). The conversion of $J(k, 0)$ in their Eq. (2.34) to fluid velocity may be obtained from Eqs. (3.1) and (3.4).

[18] One may use, for example, $\tilde{M}_{k} \equiv M_{k} / s^{4 / 3}$, provided the integral $\int \mathrm{d} k M_{k}$ is performed over comoving wave vectors. Here $s$ is radiation entropy with $s a^{3}=$ const.

[19] T. Kahniashvili, A. Brandenburg, A. G. Tevzadze and B. Ratra, Phys. Rev. D 81, 123002 (2010).

[20] K. Dolag, M. Bartelmann and H. Lesch, Astron. Astrophys. 348, 351 (1999). 